\title{
Dust acoustic wave in a strongly magnetized pair-dust plasma
}

\author{
P. K. Shukla and M. Marklund \\ Institut für Theoretische Physik IV, \\ Fakultät für Physik und Astronomie, \\ Ruhr-Universität Bochum, D-44780 Bochum, Germany
}

(Dated: Received 14 April 2004)

\begin{abstract}
The existence of the dust acoustic wave (DAW) in a strongly magnetized electron-positron (pair)dust plasma is demonstrated. In the DAW, the restoring force comes from the pressure of inertialess electrons and positrons, and the dust mass provides the inertia. The waves could be of interest in astrophysical settings such as the supernovae and pulsars, as well as in cluster explosions by intense laser beams in laboratory plasmas.
\end{abstract}

PACS numbers: 52.27.EP, 52.27.Lw, 52.35.Fp 


\section{INTRODUCTION}

The dust acoustic wave (DAW) in an unmagnetized dusty plasma was theoretically predicted by Rao, Shukla and Yu [1], which revolutionized the field of dusty plasma physics. The phase speed of the DAW is much smaller (larger) than the electron and ion (dust) thermal speed. The DAW is observed in several laboratory experiments [2, 3, 4, 5]. The properties of the DAW in a magnetized electron-ion-dust plasma has been discussed in a textbook [6].

In this paper, we consider the dispersive property of the DAW in a strongly magnetized pair-dust plasma in which electrons and positrons have equal masses. Such a pair-dust plasma can occur in supernovae and pulsar environments, as well as in cluster explosions by intense laser beams in laboratory experiments. In supernovae and around pulsars, the production of electron-positron pairs is considerable, and the magnetic field strengths can also become appreciable, especially close to the surface of neutron stars $\left(B_{\text {surf }} \sim 10^{10}-10^{13}\right.$ G). These extreme environments are well-known to host large quantities of electron-positron plasmas, as well as dust particles [7]. Clusters, on the other hand, are laboratory produced bonded atomic structures, which when irradiated by intense laser fields form plasmas on a nano-scale. These plasmas have a life span of the order of femto seconds, after which they erupt in a release of highly energetic electrons and dust particles [8, 9]. It is expected that in the next generation of lasers 10] and the free electron lasers which are under development (see e.g. [11]) will make it possible to produce electron, positrons and dust particles due to cluster explosions. Moreover, as the intense laser pulses interact with the plasma, strong magnetic fields will be generated, thus creating an environment similar to the one investigated in this paper.

The frequency (phase speed) of the DAW in our pair-dust plasma is much smaller than the electron/positron gyrofrequency (electron and positron thermal speeds). In the dust acoustic wave potential, both electrons and positrons rapidly thermalize along the magnetic field direction and establish Boltzmann distributions. Thus, pressures of inertialess electrons and positrons provide the restoring force, whereas the dust mass provides the inertia in order for the DAW to exist in a magnetized pair-dust plasma. The frequency of the DAW in a pairdust magnetoplasma is found to be larger than that in an unmagnetized electron-ion-dust plasma. 


\section{FORMULATION}

Let consider an electron-positron-dust plasma in an external magnetic field $B_{0} \hat{\mathbf{z}}$, where $B_{0}$ is the magnitude of the external magnetic field and $\hat{\mathbf{z}}$ is the unit vector along the $z$ axis. At equilibrium, we have $e n_{e 0}-q_{d} n_{d 0}=e n_{p 0}$, where $e$ is magnitude of the electron charge, $n_{j 0}$ is the unperturbed particle number density of the particle species $j$ ( $j$ equals $e$ for electrons, $p$ for positrons, and $d$ for dust grains which have the charge $q_{d}$; where $q_{d}=-e Z_{d}$ for negatively charged dust grains, $q_{d}=e Z_{d}$ for positively charged dust grains, and $Z_{d}$ is the number of charges on the dust grain surface). The perpendicular (to $\hat{\mathbf{z}}$ ) component of the electron and positron fluid velocities in the presence of low-frequency (in comparison with $\omega_{c}=e B_{0} / m c$, where $m$ is the electron/positron mass and $c$ is the speed of light in vacuum) electrostatic field $\mathbf{E}=-\nabla \phi$, where $\phi$ is the scalar potential, are

$$
\mathbf{v}_{\perp e} \simeq \frac{c}{B_{0}} \hat{\mathbf{z}} \times \nabla \phi-\frac{c T_{e}}{e B_{0} n_{e 0}} \hat{\mathbf{z}} \times \nabla n_{e 1}+\frac{c}{B_{0} \omega_{c}} \partial_{t} \nabla_{\perp} \phi
$$

and

$$
\mathbf{v}_{\perp p} \simeq \frac{c}{B_{0}} \hat{\mathbf{z}} \times \nabla \phi+\frac{c T_{p}}{e B_{0} n_{p 0}} \hat{\mathbf{z}} \times \nabla n_{p 1}-\frac{c}{B_{0} \omega_{c}} \partial_{t} \nabla_{\perp} \phi
$$

respectively, where $T_{e}\left(T_{p}\right)$ is the electron (positron) temperature and $n_{j 1}\left(\ll n_{j 0}\right.$ is a small density perturbation in the equilibrium value $n_{j 0}$. We have denoted $\partial_{t}=\partial / \partial t$. The parallel component of the electron and positron fluid velocities are obtained from

$$
\partial_{t} v_{e z}=\frac{e}{m} \partial_{z}\left(\phi-\frac{T_{e} n_{e 1}}{e n_{e 0}}\right)
$$

and

$$
\partial_{t} v_{p z}=-\frac{e}{m} \partial_{z}\left(\phi+\frac{T_{p} n_{p 1}}{e n_{p 0}}\right)
$$

Inserting Eqs. (11) and (2) into the electron and positron continuity equations and assuming that $\left|\partial_{t}\right| \ll V_{T e, T p}\left|\partial_{z}\right|, \omega_{c}\left|\partial_{z}\right|$, we obtain

$$
n_{e 1} \simeq \frac{n_{e 0} e \phi}{T_{e}} \text { and } n_{p 1} \simeq-\frac{n_{p 0} e \phi}{T_{p}}
$$

which indicate that both electrons and positrons will thermalize rapidly along the magnetic field direction $\hat{\mathbf{z}}$, and establish Boltzmann distributions. Here $V_{T e}=\left(T_{e} / m\right)^{1 / 2}$ and $V_{T p}=$ $\left(T_{p} / m\right)^{1 / 2}$ are the electron and positron thermal speeds, respectively. 
We are interested in the DAW whose frequency is much larger than the dust gyrofrequency. Thus, charged dust grains are unmagnetized and their dynamics is governed by the dust continuity and momentum equations

$$
\partial_{t} n_{d 1}+n_{d 0} \nabla \cdot \mathbf{v}_{d}=0
$$

and

$$
\partial_{t} \mathbf{v}_{d}=-\frac{q_{d}}{m_{d}} \nabla \phi
$$

where $m_{d}$ is the dust mass. Equations (33)-([5) are closed by means of Poisson's equation

$$
\nabla^{2} \phi=4 \pi\left(e n_{e 1}-q_{d} n_{d 1}-e n_{p 1}\right)
$$

which takes the form

$$
\left(\nabla^{2}-k_{D}^{2}\right) \phi=-4 \pi q_{d} n_{d 1},
$$

using Eq. (3) . Here $k_{D}^{2}=\left(1 / \lambda_{D e}^{2}\right)+\left(1 / \lambda_{D p}^{2}\right)$, with $\lambda_{D e}=\left(T_{e} / 4 \pi n_{e 0} e^{2}\right)^{1 / 2}$ and $\lambda_{D p}=$ $\left(T_{p} / 4 \pi n_{p 0} e^{2}\right)^{1 / 2}$ being the electron and positron Debye radius, respectively.

Combining (41) and (51) we have

$$
\partial_{t}^{2} n_{d 1}=\frac{n_{d 0} q_{d}}{m_{d}} \nabla^{2} \phi
$$

Substituting for the Laplacian of the potential using Eq. (6), and eliminating $n_{e, p 1}$ by means of (3) we have

$$
\left(\partial_{t}^{2}+\omega_{p d}^{2}\right) n_{d 1}=\frac{\omega_{p d}^{2} k_{D}^{2}}{4 \pi q_{d}} \phi,
$$

where $\omega_{p d}=\left(4 \pi n_{d 0} q_{d}^{2} / m_{d}\right)^{1 / 2}$ is the dust plasma frequency.

Supposing that $\phi$ and $n_{d 1}$ is proportional to $\exp (i \mathbf{k} \cdot \mathbf{r}-i \omega t)$, where $\mathbf{k}$ and $\omega$ are the wavevector and frequency, Eqs. (17) and (9) yield

$$
\omega=\frac{k C_{D}}{\left(1+k^{2} \lambda_{D}^{2}\right)^{1 / 2}},
$$

which is the dust acoustic wave frequency in a strongly magnetized pair plasma. Here $C_{D}=\omega_{p d} \lambda_{D}$ is the dust acoustic speed and $\lambda_{D}=\left[\lambda_{D e} \lambda_{D p} /\left(\lambda_{D e}^{2}+\lambda_{D p}^{2}\right)\right]^{1 / 2}$ is the effective pair plasma Debye radius. We note the resemblance between the frequency spectrum (12) of the DAW in an electron-positron-dust with that DAW in an unmagnetized electron-iondust plasma. In the latter with $T_{e} \gg T_{i}$ and $n_{i 0}>n_{e 0}$, we have $\lambda_{D}=\lambda_{D i} \ll \lambda_{D e}$, where $\lambda_{D i}=\left(T_{i} / 4 \pi n_{i 0} e^{2}\right)^{1 / 2}$ is the ion Debye radius. On the other hand, in an electron-positron-ion 
plasma, we typically have $\lambda_{D}=\lambda_{D e} /(1+\sigma)^{1 / 2}$, where $\sigma=T_{e} n_{p 0} / T_{p} n_{e 0}$. Thus, the dispersion properties of the DAW in a magnetized pair-dust plasma are significantly different from those in an unmagnetized electron-ion-dust plasma.

\section{SUMMARY}

In summary, we have examined the linear property of intermediate frequency, viz. $\omega_{c d}=$ $\left|q_{d}\right| B_{0} / m_{d} c \ll \omega \ll \omega_{c}$, DAW in a strongly magnetized pair-dust plasma. The DAWs are supported by the pressure of the inertialess electrons and positrons, whereas the mass of the dust grains provide the inertia.

The results presented in this paper should be of importance in the application of the next generation lasers to cluster irradiation, and the ensuing cluster explosion. These events are likely to produce an environment in which pair plasmas, strong magnetic fields, and dust particles coexist. Similarly, in supernovae, the pair plasma will inevitably contain dust particles, and will be interpenetrated by magnetic fields, thus creating physical conditions at which the DAW derived in this paper will occur.

\section{Acknowledgments}

The authors dedicate this paper to Lennart Stenflo on the occasion of his 65th birthday.

This work was partially supported by the European Commission (Brussels, Belgium) through contract No. HPRN-CT-2001-00e14 for carrying out the task of the Human Potential Research Training Networks "Turbulent Boundary Layer", as well as by the Deutsche Forschungsgemeinschaft (Bonn, Germany) through the Sonderforschungsbereich 591 and by DOE Grant No DE-FG03-97ER54444.

[1] N. N. Rao, P. K. Shukla, and M. Y. Yu, Planet. Space Sci. 38, 534 (1989); P. K. Shukla, Phys. Plasmas 8, 1791 (2001); ibid. 10, 1619 (2003).

[2] A. Barkan, R. L. Merlino and N. D’Angelo, Phys. Plasmas 2, 3563 (1995); R. L. Merlino, A. Barkan, C. Thompson, and N. D'Angelo, ibid 5, 1607 (1998).

[3] H. R. Prabhakara and V. L. Tanna, Phys. Plasmas 3, 3176 (1996). 
[4] V. I. Molotkov, A. P. Nefedov, V. M. Torchinskii et al., JETP 89, 477 (1999).

[5] N. Hayashi, Phys. Plasmas 8, 3051 (2001).

[6] P. K. Shukla and A. A. Mamun, Introduction to Dusty Plasma Physics, Institute of Physics, Bristol, 2002.

[7] T. Tajima and K. Shibata, Plasma Astrophysics (Addison Wesley 1997).

[8] T. Ditmire, J. W. G. Tisch, E. Springate, et al., Nature 386, 54 (1997); T. Ditmire, J. W. G. Tisch, E. Springate, et al., Phys. Rev. Lett. 78, 2732 (1997).

[9] C. S. Liu and V. K. Tripathi, Phys. Plasmas 10, 4085 (2003).

[10] G.A. Mourou, C.P.J. Barty and M.D. Perry, Phys. Today 5122 (1998).

[11] URL http://www-hasylab.desy.de/facility/fel/ 